\title{
Statistical Interaction Modeling of Bovine Herd Behaviors
}

\author{
Bruce Stephen, Member, IEEE, Cathy Dwyer, Jimmy Hyslop, Matthew Bell, David Ross, Kae Hsiang \\ Kwong, Craig Michie and Ivan Andonovic, Senior Member, IEEE
}

\begin{abstract}
While there has been interest in modeling the group behavior of herds or flocks, much of this work has focused on simulating their collective spatial motion patterns which have not accounted for individuality in the herd and instead assume a homogenized role for all members or sub-groups of the herd. Animal behavior experts have noted that domestic animals exhibit behaviors that are indicative of social hierarchy: leader/follower type behaviors are present as well as dominance and subordination, aggression and rank order, and specific social affiliations may also exist. Both wild and domestic cattle are social species, and group behaviors are likely to be influenced by the expression of specific social interactions. In this paper, Global Positioning System coordinate fixes gathered from a herd of beef cows tracked in open fields over several days at a time are utilized to learn a model that focuses on the interactions within the herd as well as its overall movement. Using these data in this way explores the validity of existing group behavior models against actual herding behaviors. Domain knowledge, location geography and human observations, are utilized to explain the causes of these deviations from this idealized behavior.
\end{abstract}

Index Terms-Agriculture, Behavioral science, Global Positioning System, Markov processes

\section{INTRODUCTION}

$\mathrm{D}$ OMESTIC cattle are known to possess social traits which induce different interactions with the other animals in their herd. Animal behaviorists have long noted the connection between physiology and social activity which emphasizes the importance of increasing understanding of the mechanisms that underlie grouping behavior. Although prior research has produced simulations of herding behavior that approximates collective behaviors this has been in an oversimplified manner that adopts a generic behavior for all animals. The reality is far removed from this homogeneity, with a variety of dominant, subservient and isolationist social traits having been observed by behavioral experts. In a review of cattle and domestic

Manuscript received November $30^{\text {th }}, 2009$. This work was supported in part by ITI Techmedia as part of the Condition Based Monitoring Programme.

Dr. B. Stephen is a Research Fellow in the Advanced Electrical Systems Research Group, Institute of Energy and Environment, University of Strathclyde, Glasgow, G1 1XW (phone: +44 (0)141 548 5864, e-mail: bstephen@eee.strath.ac.uk)

Dr. C. Dwyer, Dr J. Hyslop, Mr. D. Ross and Mr. M. Bell are with Scottish Agricultural College, King's Buildings, West Mains Road, Edinburgh, EH9 $3 \mathrm{JG}$. animal social characteristics, [1] noted that herds exhibited fairly complex social interactions that were indicative of dominance and subordination with clear leaders and followers along with evidence of care and dependency. As an example of how activities of individuals can impact on the welfare of others, [2] noted that groupings of animals diluted their predatory risk and improved their foraging ability confirming that the existence of reactionary, agonistic and learning behaviors will motivate the herd to either respond to or imitate the actions of an individual. Domesticated animals also show differences in social behavior, including both agonistic responses (butting, pushing, avoidance) and affiliative behaviors [3]. While environmental and physiological factors may affect behavior, there remains evidence of a social order within herds; dominant animals are noted to defend territory with age and weight correlating with herd rank [1].

In a management context, understanding and being able to quantify aspects of an animal's behavior may increase the ability to manage the animal for both economic productivity and animal welfare reasons. Modeling GPS based positional datasets on parameters such as displacements, spatial distribution and activity levels are an innovative way to gain new insights into both individual animal and herd or group behaviors. Understanding animal behaviors such as grazing patterns, feeding or rumination time budgets and animal to animal social interactions will assist our ability to manage grazing provision, mating strategies and disease epidemiology patterns across and within herds.

Formulating a model of herd behavior that captures all of its complexities has been attempted in several ways with simulations of simple pseudo-autonomous creatures and models derived from geospatial data being at either extreme of the research spectrum; this paper attempts unification between these disparate strands of research by learning a model from observed herd activities and relating its inference to hypothetical behavior constructs used for group simulation. Utilizing a set of GPS transponder data gathered in continuous periods over several weeks from a free ranging herd of beef cows, a Markov Random Field model is created, through the choice of an appropriate neighborhood system that automatically segments the herd at each timeframe, labeling animals according to their relationships with others.

This paper is structured as follows: in the next section, the existing strategies for modeling herd behavior are reviewed 
with particular emphasis being placed on the pioneering concepts of behaviour predicate based modelling within the context of a group and also more recent studies involving animals equipped with GPS telemetry. Although the methodology for investigating herding behavior presented here is data driven, how the model relates to what is observed is of primary importance from a ground truthing perspective and drives several of the design decisions such as feature selection and neighbourhood function. In section 3 , this modeling strategy is outlined, covering the collection of exemplar herd data from free ranging beef cattle and the choice of features necessary to represent herding behavior. Section 4 reviews Markov Random Fields and their derivatives and the specializations required for adapting them to herd modeling are shown. After this, the algorithmic details used to apply the model are presented along with the system implemented to visualize the collective and individual behaviors present in the herd over time. Finally, extensions to the current model and further development required for practical applications are described in the conclusions.

\section{Prior Group Behavior Modeling StrategIES}

Among the earliest attempts to spatially simulate herding animals was a model of group behaviors based on steering and separation known as 'boids' [4]. These assumed uniform attraction and repulsion forces throughout the group with no incorporation of individuality; while in reality, some animals will be sociable and others less interested in the rest of their group. An additional level of autonomy can be encoded in the spatial models, an example being [5] where a herd of simulated 'robots' of point mass were used to demonstrate how simple group behavior could be regardless of the complexity of the individual; this contribution attempted to simulate a herd through consideration of motion primitives applied by the individual. Unlike the earlier herding models, there were more layers of detail: a perception model was incorporated which determined the animals in the herd that were visible within a given radius; a placement algorithm was used to determine the desired relative position; lastly, a simple spring/damper model control system was employed for computing the velocity of the animal within the constraints of the position and velocity of its immediate neighbors. However, all animals still had the same dynamics and had them all of the time. A further development in group behavior models, made by [6], programmed in individuality with 'autonomous characters', proposing multilayer architecture of action selection, steering and locomotion much like [5]. However, key to this was a set of basic steering behaviors such as 'seek', 'flee' and 'evasion' that dictated a characters placement, velocity and directional bearing based on encounters with other characters - behavior of the individual was no longer homogenous, it could change to reflect circumstance.

Rather than imposing these behaviors, these could be learned, as in [7], where hypothetical behavior state based models derived from Markov chain variants were learned.
Robots were programmed using a behavior predicate based language, whose predicates were not dissimilar to the steering behaviors in [6], actions from subsequent robot operations could then be used to learn a model, mapping the states of the Markov chain to a subset of the behavior predicates. Similarity in behavior between robots could be evaluated by comparing the resultant likelihoods of a model generating a given task sequence.

A solution to obtaining a less regimented picture of herding behavior is to consider a real herd of animals. With recent advances in low cost sensor technology, a number of applications of GPS transponders to herding animals have attempted to capture behavior to previously unknown levels of detail. Such studies had the potential to validate earlier theories of grouping behavior and elaborate on their key features. In [8], a trial of GPS telemetry mounted on wild Zebra was described, tracking their mobility characteristics over a $20 \mathrm{~km}$ square range and noted that several behavior mechanisms were present resulting in three different mobility regimes being identified. [9] monitored daily activities of Zebu cows in Western Niger using GPS in tandem with observer's records to build a classifier of activity from movement. The study used behavioral expert observations to verify classification of walking, resting and grazing bouts from 10 second GPS fixes. These resulted in daily activity budgets composed of proportions of these 3 behaviors which differed significantly depending on time of day. Using a small herd of Hereford breed beef cattle, [10] trained cluster (specifically, K-Means [11]) models on free-ranging cow behaviors to classify active and inactive states. Equipment that measured head angle via an inclinometer and magnetometer was used along with recordings of GPS fixes; classifications of these were then coupled with biological observations to infer the meaning of the clusters and found, in particular, that low speed and high head angle were deemed traits of inactivity while high speed and low head angle were traits of an active animal. Mean cow distance to herd centroid and standard deviation showed that cows are closer during activity than inactivity. Activity levels were shown to mutually influence each other among gregarious animals. The continuing increases in reliability and affordability of sensors led to more practical applications of identifying behavior states. Although not involving a model of herding animals, [12] used GPS measurements to implement a 'virtual electric fence' in real time to keep bulls separated without farm staff intervention. This pair-wise interaction model proposed used separation distances and closing velocity vector alignment to detect a converging bull. The model was based on a finite state machine with transition parameters derived from threshold values of input features obtained through experimentation. Learning, rather than manually specifying a multi-behavior regime model, in [13], an earlier dynamic model was extended to incorporate multiple behavior regimes: stressed and grazing. The model hypothesizes that the social characteristics of individuals will alter according to their active behavior state. 
Using positions and derived component velocities, the dynamic model evaluates an 'agent interaction force' as a measure of an animal's gregariousness.

\section{Construction of A Herd Behavior Model}

Construction of a model of group behavior that captures the type of discrete interaction activities that [5] identified but without generalizing this type of behavior to an individual requires three key elements. The first is a set of exemplary behaviors from a real herd to demonstrate how the members of a group behave towards each other and how this changes depending on the presence of others. The second is to identify the features of this set that characterize the behavior of interest. The final element is to capture the particular values of the features or combinations of values that recur on a regular basis and do this in a manner that is robust to noise.

\section{A. Exemplar Herd Behavior}

The behavior of a group of animals was captured as GPS fixes over time. 14 animals were equipped with collar mounted GPS transponders manufactured by Bluesky Telemetry Ltd of Aberfeldy, Scotland, shown in figure 1, which were configured to record fixes every 3 minutes and periodically transmit this data wirelessly to be ultimately accrued in a relational database.

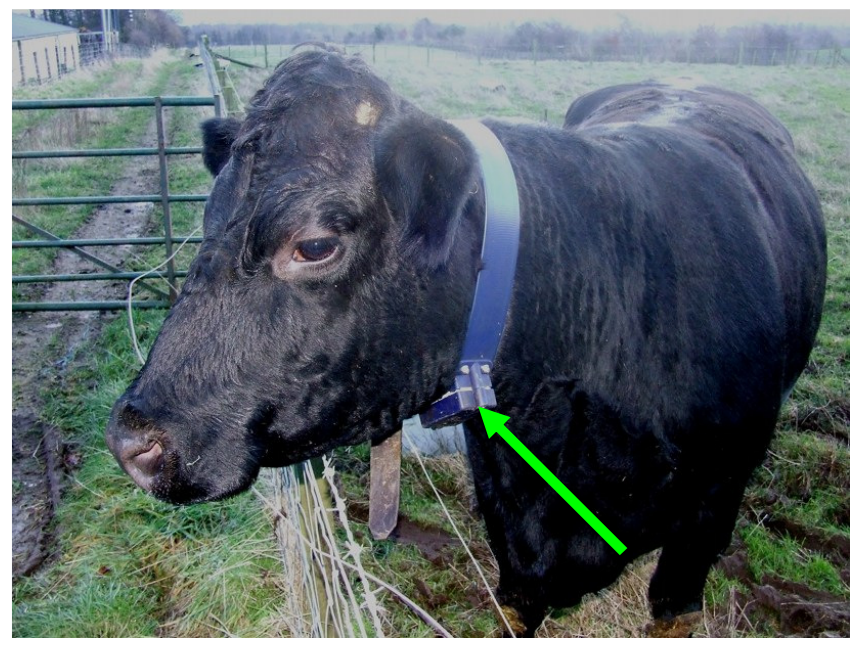

Fig. 1. Beef cow wearing a collar mounted GPS transponder.

The database was populated between June 2006 and September 2006, with runs of up to 7 days which gave 946 whole cow days. The animals studied were spring calving beef cows that had all calved at least once and were grazed as part of a small herd during the months of June - September 2006. Cows and their suckling calves were rotationally grazed in one of two fields of 4.4 and 6.2 hectares (ha) respectively during this period at stocking rates of approximately 1.5 livestock units/ha; typical of commercial farming practice in Scotland. The location was on the eastern mainland of Scotland, UK and the altitude of the fields was approximately $210 \mathrm{~m}$ above sea level. In order to utilize the GPS data, established procedures [14] are used to convert the geodetic latitude, longitude and altitude into their Cartesian equivalents at each time t. All GPS estimates of position have some degree of error in them. The extent of this error cannot be accurately estimated but is known to be inversely proportional to the number of satellites used to produce the fix. In [15] the relationship between the numbers of satellites and the impact this had on position accuracy was noted.

\section{B. Characterizing Herding Features from Geospatial Data}

To best represent animal behavior with respect to the rest of the herd and its immediate neighbors, more than just the position must be considered. Features derived from GPS data must be able to capture elements of animal to animal social interactions that are significant to the animals welfare and management. These would include individual and total within herd contacts, movements with the herd as a group (or subgroups within the herd) and the degree of "solitude" or lack of contact. All of these elements may differ widely according to grazing availability, breed type, position within the social herd hierarchy and previous learned experience.

Animals in a herd of size $H$ are represented by a point $\mathrm{c}$ in 2-dimensional space at time $t$. To track the motion of the herd as a group, a useful quantity is the herd centroid $g$. To contextualize the distance between cows, the dispersal $d$ of the herd is also used which amounts to the average distance from the centroid. These two quantities indicate where most animals are positioned and how tightly they are assembled. For a herd of size $H$, there will be a symmetric $\mathrm{H}$ by $\mathrm{H}$ inter-cow distance matrix B, cells of which measure the distance between any 2 cows. This is used to find the animals closest to a given cow normalized using the herd dispersal. GPS collars are not synchronized, so to ensure that there is a representation of all animals at all time steps, a state machine approach to preprocesses the position data extracted from the database is used to calculate the herd dispersal, centroid and inter-cow distance matrix from accumulated fixes. Position and spacing are able to represent static grouping behaviors such as cohesion or solitude, but dynamic behaviors such as pursuit or evasion require temporal and directional observations. Figure 2 shows the bearing and displacement for a single animal over a 24 hour period. This illustrates how a single animal can exhibit varying levels of activity through its displacements, and deliberation through its changes in heading with respect to the herd. Aside from providing a context for the level of activity of individuals, on its own the group displacement remains a

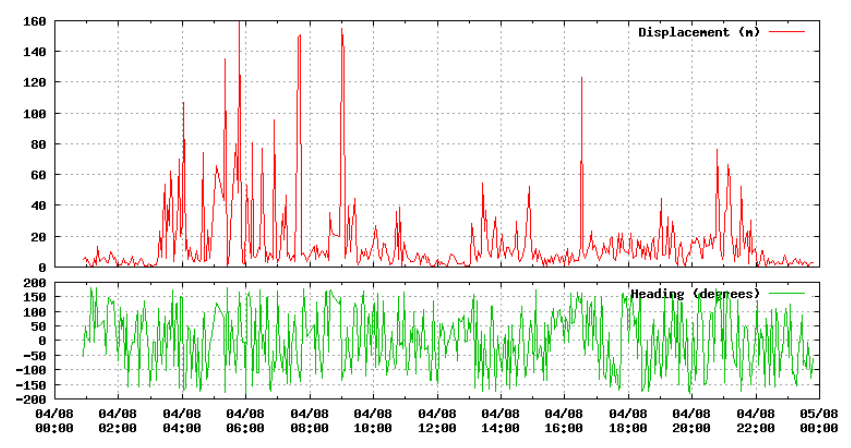

Fig. 2. Illustration of the changes in displacement and bearing for a single animal over 24 hours. 
useful indicator: activity of the group as a whole may be suppressed by hot weather or excited by the arrival of feedstock. The cows themselves are assumed to be stateless with no memory of their previous activities. While this is questionable in reality, the temporal independence assumption is replaced by one of dependence on the group.

\section{MARKOV RANDOM FIELDS}

There are two problems inherent in modeling herding behaviors: firstly, the general behavior of individuals needs to be captured and abstracted in such a way that it is robust to imprecise observations; secondly, the interaction between individuals needs a similarly generalized representation to be learned. The preferred means of capturing such dynamics while retaining robustness to noise is through the statistical modeling of the variables in the system. 'Cluster' type models such as K-Means [11] or Finite Mixture Models [11, 16] that abstract a multivariate, continuous observation into a single discrete label or state are based on the assumption that there are subpopulations in the observation data generated by processes with differing statistical distributions. However, such models are not immediately suited to capturing complex dependencies between variables, as individual observations are attributed to generator processes that are assumed independent of one another. The notions of underlying or hidden generator $[17,18]$ processes stem from more basic latent class models [19] where observed variables are implicit of some abstraction or state label; collections of generators form particular configuration architectures with the configuration being defined by the bonds or interfaces between generators and the state of each generator. This model serves as a formalism of structural or 'closed' patterns [18], a structural familiarity that simplified observations by finding regularities in their constituent parts rather than the whole.

\section{A. Graphical Models}

A convenient way of representing the probabilistic relationships between these processes is by using graphical models [20]. The models feature nodes representing random variables that, if statistically dependent, are interconnected with arcs representing this relationship. In some graphical models, these arcs are directed to imply causality; however there are circumstances where undirected arcs are preferred. It may be unclear whether one variable 'causes' another or it may just be the case that causal structure is irrelevant and all that matters is the co-occurrence of variables. The tractability of graphical models is only realizable through the adoption of the Markov assumption which restricts the scope of influence variables have on each other. Like a multidimensional generalization of the order of a Markov chain, a Markov blanket represents the extent of dependence on a particular variable. For spatial data, the Markov blanket extends in 2dimensions leading to an undirected graph known as a Markov Random Field (MRF). An MRF is typically used to obtain the global effect of local relationships such as the interpretation of images from pixel arrangements or the classification of characters from assemblies of strokes [21]. From the
Hammersley Clifford Theorem, an MRF is distinguished from a Random Field by the fact that the state variables $q$ in a configuration are distributed according to a Gibbs distribution:

$$
P(q)=\frac{1}{Z} \exp \{-U(q)\}
$$

Where $\mathrm{Z}$ is the normalizing constant given by summing over all possible observations for $\mathrm{q}$ in the graph:

$$
Z=\sum_{q \in Q} \exp \{-U(q)\}
$$

$\mathrm{U}$ is an energy function that is strictly positive and is chosen according to how configurations are defined in the model application. The energy of a configuration is usually obtained by evaluating all clique potentials $\mathrm{V}$ over all connected sites. A potential function $\mathrm{V}$ is an arbitrary function used to measure the relationship between variables or multiple variables in the clique $\mathrm{c}$, the definition of this can be application specific. Cliques are subsets of the graph containing connected variables. As an example consider the graph representation of variables in a regular lattice arrangement shown in figure 3.

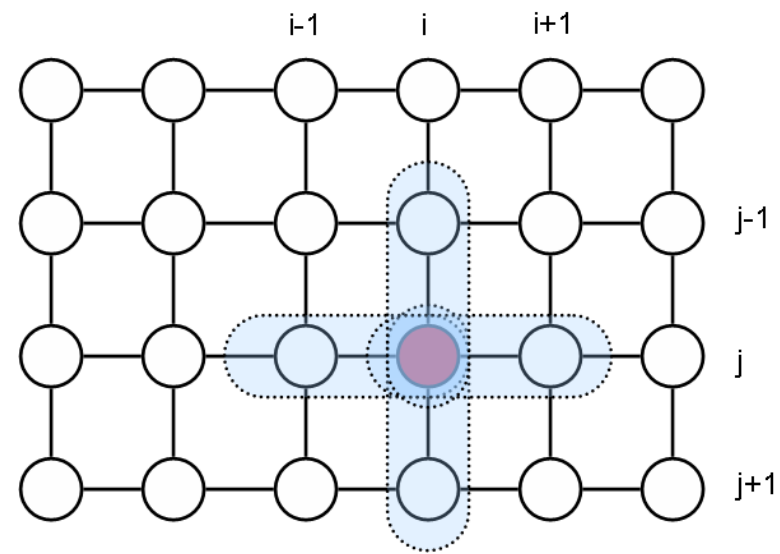

Fig. 3. A lattice shaped random field with a simple neighborhood formed from adjacent nodes. The shaded areas represent cliques of the red site.

This type of graph is common in image analysis applications where each node represents a pixel whose value may be dependent on at least one of its neighbors, hence the connection structure. The clique size and therefore perceived influence is limited by a function called a neighborhood system, $\mathrm{N}$, which dictates the variables that can be included in a clique for a site; in the example in figure 3 it is each variable that has an adjacent connected variable in both directions on either side of it. The neighborhood of the site at $i, j$ contains 4 cliques. The relationship of all cliques at all sites, I, forms the joint density over all variables in the graph. Cliques come in various orders; an $n^{\text {th }}$ order clique contains $n$ connected sites all of which must form part of the neighborhood of the configuration being considered. A scheme used by [21] in character recognition and [23] in image segmentation is to use $1^{\text {st }}$ and $2^{\text {nd }}$ order clique potentials:

$$
U(q)=\sum_{i \in I} V_{1}\left(q_{i}\right)-\sum_{j \in \mathrm{N}_{i}} V_{2}\left(q_{i}, q_{j}\right)
$$

Although higher order cliques are possible, $1^{\text {st }}$ and $2^{\text {nd }}$ order capture statistical observation details about the site, how the 
site is labeled, from the first order potential function and structural information, how the sites interact, from the second order one. This way, the MRF captures localized dependencies without requiring a complex conditional dependency structure for the entire graph - a key part of the 'recognition by components' theory of perception [18].

\section{B. Hidden Markov Random Fields}

In situations where the observation is assumed to be noisy and/or must be abstracted into one of a finite number of states that represents a generalization of its value, the MRF can still be used to model the dependency structure of the graph. However, since the state labels are never observed but instead are implied by the observation, a different model called a Hidden Markov Random Field (HMRF) is required. The MRF works as before but an additional layer of inference is required to map the observation or emission field onto the corresponding locations in the graph. For an HMRF the joint distribution of the hidden $(\mathrm{Q})$ and observed $(\mathrm{O})$ variables $\mathrm{P}(\mathrm{Q}$, O) is required:

$$
P(q, o)=\frac{1}{Z} \exp \{-U(q, o)\}
$$

In this case the energy function is of both the observed and hidden variables although the choice of clique potentials in $U$ are made to reflect preferred configurations of site labels implied by observations: likely configurations are still required to have the lowest energy but for an HMRF, the energy function is now a function of both the state and the observation:

$$
U(q, o)=\sum_{i \in I} V_{1}\left(o_{i} \mid q_{i}\right)-\sum_{j \in \mathrm{N}_{i}} V_{2}\left(q_{i}, q_{j}\right)
$$

The observed random variables are assumed to be conditionally independent with the dependency structure captured through the hidden variables. For an observation o, a function is required to relate the observations back to the configuration of the underlying MRF states that generated it.

\section{Herd Model Specialization of HMRF}

There are two parts to the HMRF that are domain specific, the neighborhood function and the clique potentials that are used in the energy function. This section discusses how these are formulated for the herd application. While Hidden Markov Random Fields have been extensively employed in pattern analysis tasks, these have mainly focused on imaging data where the regular structure of pixels lends itself to simple neighborhood functions based on site adjacency like that shown in figure 3. However, there is no concept of spatial adjacency in the herding application as firstly the herd is not regularly aligned in space like lattice data and second, the animals are mobile causing the neighboring cows for a given site (cow) to change. Instead, this neighborhood will be concerned with the implicit influence of variables rather than their position so the herd neighborhood will be constructed according to the proximity of other cows. The neighborhood function proposed for the herd model is a simple maximization of the inter-cow distance matrix at a given time step. For every cow this forms a neighborhood from the $\mathrm{K}$ nearest cows.

If $\mathrm{K}$ was set to $\mathrm{H}$ then the assumption would be that the entire herd influences each individual which assumes an unrealistic degree of awareness; if $\mathrm{K}$ was set to 1 then it would be assumed that a cow was influenced only by its nearest neighbor which would return to the over simplistic herd representation. Neighborhoods consisting of the 3 or more nearest cows are considered, as this will go beyond a single pair-wise interaction. Selecting the ideal neighborhood size is a model selection problem dealt with in section VB. In figure 4 , the sites (cows) in three neighborhoods are highlighted with a filled shape and the cows in the neighborhood enclosed. Note how only part of the herd is in a particular cow's neighborhood - this is more realistic and follows the thinking of the real herd models and herd-animals actual awareness of the rest of the herd i.e. there is going to be a limit to the influence of distant animals.

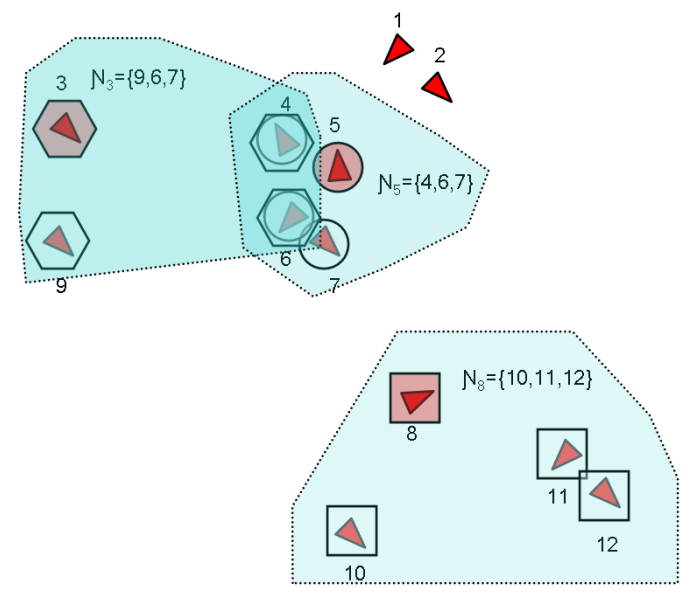

Fig. 4. Neighborhoods formed for animals 3, 5 and 8. Neighborhoods for the remaining animals are not shown to preserve clarity. Animals can belong to multiple neighborhoods and will change neighborhood over time.

Defining the neighborhood function leaves the energy function definition as the remaining specialization. The energy function must be defined to prefer recurring combinations of arrangements of cow behavior, that is, cows behaving in a particular way are observed with cows behaving in a (or possibly another) particular way. This is achieved by expressing the first order clique, which captures single animal behavior, as a collection of independent Gaussian distributions, then weighting these with a connectivity matrix which forms the basis of the second order clique and captures behavioral ensembles in the herd. Since the minimization of this function is required for preferred configurations, the log of these is used. Each state observation is assumed to be Gaussian distributed so taking the negative log of this, gives a function that decreases with increasing observation likelihood: 


$$
\begin{aligned}
& V_{1}\left(o_{i}(t) \mid q_{i}=k\right)= \\
& -\log \left[\frac{1}{\sqrt{2 \pi\left|\Sigma_{k}\right|}} \exp \left\{-\frac{1}{2}\left(o_{i}(t)-\mu_{k}\right)^{T} \Sigma_{k}^{-1}\left(o_{i}(t)-\mu_{k}\right)\right\}\right]
\end{aligned}
$$

Each state has its own mean and elliptic covariance matrix, the latter to relax dependence assumptions between observation features. For the second order clique potential, the cooccurrence of states is used:

$$
V_{2}\left(q_{i}, q_{j}\right)=-\log a_{i j}
$$

The element $a_{i j}$ of the matrix $A$ is the probability of the label $j$ being seen as a neighbor of a site labeled $i$ - which is analogous to the likelihood of one cow exhibiting a particular type of behavior being seen with a cow exhibiting another particular behavior type. To reiterate, the first order clique sets the most likely state the cow is in with respect to its observed behavior. The second order clique reduces the energy of the model if the cow state is likely within the context of its neighbor's states.

\section{APPLYING THE MODEL}

Table I lists the animals used in the trials which were a mixture of ages, sizes and breeds. These were all either from the Aberdeen Angus (AA) or Limosin (LIM) breeds. While age was not recorded, parity, the number of times a cow has calved provides a measure in proportion to how old the animal is. Similarly, weight gives an approximation of size; both parameters may be useful in explaining interactions such as dominance where size (physical stature) and age may be influencing factors in the roles played within the herd.

TABLE I

ANIMALS USED IN BEHAVIOR TRIAL

\begin{tabular}{cccc}
\multicolumn{4}{c}{ ANIMALS USED IN BEHAVIOR TRIAL } \\
\hline \hline Identifier & Breed & Weight $(\mathrm{kg})$ & Parity \\
& & & \\
LX1 & LIMx* & 720 & 5 \\
AX1 & AAx & 748 & 5 \\
LX2 & LIMx & 568 & 2 \\
LX3 & LIMx & 568 & 2 \\
LX4 & LIMx & 686 & 3 \\
AX2 & AAx & 612 & 2 \\
AX3 & AAx & 720 & 4 \\
AX4 & AAx & 642 & 2 \\
AX5 & AAx & 710 & 8 \\
LX5 & LIMx & 640 & 6 \\
AX6 & AAx & 682 & 6 \\
AX7 & AAx & 706 & 6 \\
LX6 & LIMx & 798 & 6 \\
AX8 & AAx & 770 & 5 \\
AX9 & AAx & 748 & 4 \\
& & & \\
\hline \hline
\end{tabular}

†Parity refers to the number of times the animal has been in calf.

*Letters denote the following breeds LIMx = Limousin cross-bred, AAx = Aberdeen Angus cross-bred.

\section{A. Estimation of Model Parameters}

The distributions that relate states to observations and the connectivity matrix must be learned from a set of exemplar data. In [21] a Maximum Likelihood implementation of the Expectation Maximization (EM) algorithm [22] was used to learn an HMRF. EM is an iterative procedure that, for maximum likelihood estimation, computes the distribution of the state variables with respect to the observed data then chooses model parameters such that this likelihood is maximized. The algorithm continues iterating through the two steps until the log likelihood of the data converges.

[11] provided the following general view of the EM algorithm used to estimate a parameter $\theta$ using a set of observed data $\mathrm{O}$ with unobserved data $\mathrm{Q}$ :

1. Estimate $\theta$ as $\hat{\theta}$

2. On the Expectation step calculate $P(Q \mid O, \hat{\theta})$

3. Calculate:

$$
Z(\theta, \hat{\theta})=\sum_{Q} P(q \mid O, \hat{\theta}) \log P(O, q \mid \hat{\theta})
$$

From which the maximization of $\mathrm{Z}$ gives the parameter update: $\theta=\underset{\theta}{\arg \max } Z(\theta, \hat{\theta})$

4. If $Z$ has not converged, set $\hat{\theta}=\theta$ and repeat from the Expectation step (2).

For the Gaussian observation models used in the first order cliques, the K-Means [11] clustering algorithm is used to first find approximations of feature means and variances. Using these, the connectivity matrix is filled in with counts of cooccurring clusters and then normalized. For an HMRF, no closed form solution for the Expectation step exists, so the state labeling must be obtained by other means:

$$
\max _{k} P\left(q_{i}=k \mid o_{i}(t)\right)
$$

A common choice of algorithm for this task is Iterated Conditional Modes (ICM) [23, 24] which implicitly minimizes the energy function by maximizing the posterior density energy function given by (8), sequentially at each site through the choice of site labels at each time frame. The maximization step is a straightforward maximum likelihood estimate akin to that for a finite mixture model [16].

The beef cows used were kept outside during the duration of the trial and for days chosen for analysis, consequently had no predictable routine outside of a daily visual check by farm and technical staff. Given this limit of distractions, data over a single 24 hour period is used to train the model resulting in 5325 fixes or 408 frames. The model typically converges after around 20 iterations of EM. 


\section{B. Social behaviors as state labels}

The cardinality of $\mathrm{Q}$ is another variable quantity in the model: as this effectively abstracts recurring feature vector values, the states can be construed as particular behaviors. The number of behaviors assumed present in the data must be specified prior to starting training.

Many MRF models have a binary state variable, however, multivariate states are known in image processing and texture analysis applications of HMRFs to allow different underlying abstractions to be represented. The same philosophy prompts the choice of multiple states in this model; firstly it allows a more complex emission distribution to be learned and in doing so allows a range of implied group behavior patterns to be revealed. This application assumes that states and configurations of an MRF will correspond to particular group behavior which can be interpreted through the parameters of the emission distribution associated with each state.

To select the optimal number of behavior states, the model most likely to have generated the data is used. This maximum likelihood approach to model selection has the disadvantage that it finds every possible behavior at the expense of loss of generality of the model. Several model selection criteria such as Bayesian Information Criteria, Normalized Entropy Criterion, Automatic Relevance Determination and Akaike Information Criterion [11, 16] could be employed to lessen this effect although with an increase in computational overhead.

\section{Labeling Animals by Interactions}

Once an MRF has been trained it can be used to find the optimal label structure of the herd given a set of observations, much in the same way the Viterbi algorithm is used to find the implied state sequence in a Hidden Markov Model (HMM). Unlike the trellis structure of possible sequence likelihoods in an HMM, the state of the herd is retained in a frame generated at each time step by the herd state machine. This 'frame' is an irregular structure that holds the observation feature vector for each animal as well as its state label and pointers to the animals captured by its neighborhood function. Another problem is that unlike the trellis, the graph structure of the MRF model does not permit closed form inference, a problem encountered in the formulation of the Expectation step for parameter estimation. Again, the ICM algorithm [23, 24] is employed to approximate the site labeling by maximizing the probability at each site with respect to the observation by piecewise re-labeling of the neighboring sites. Obviously, changing the labeling at one site will impact on the others which share it in their clique, so this optimization must be performed globally by traversing the entire graph until the global energy converges. In an image processing application, this algorithm would traverse the data set in a raster fashion, however, as already noted, this procedure is further complicated by the herd fixes not having this regularized

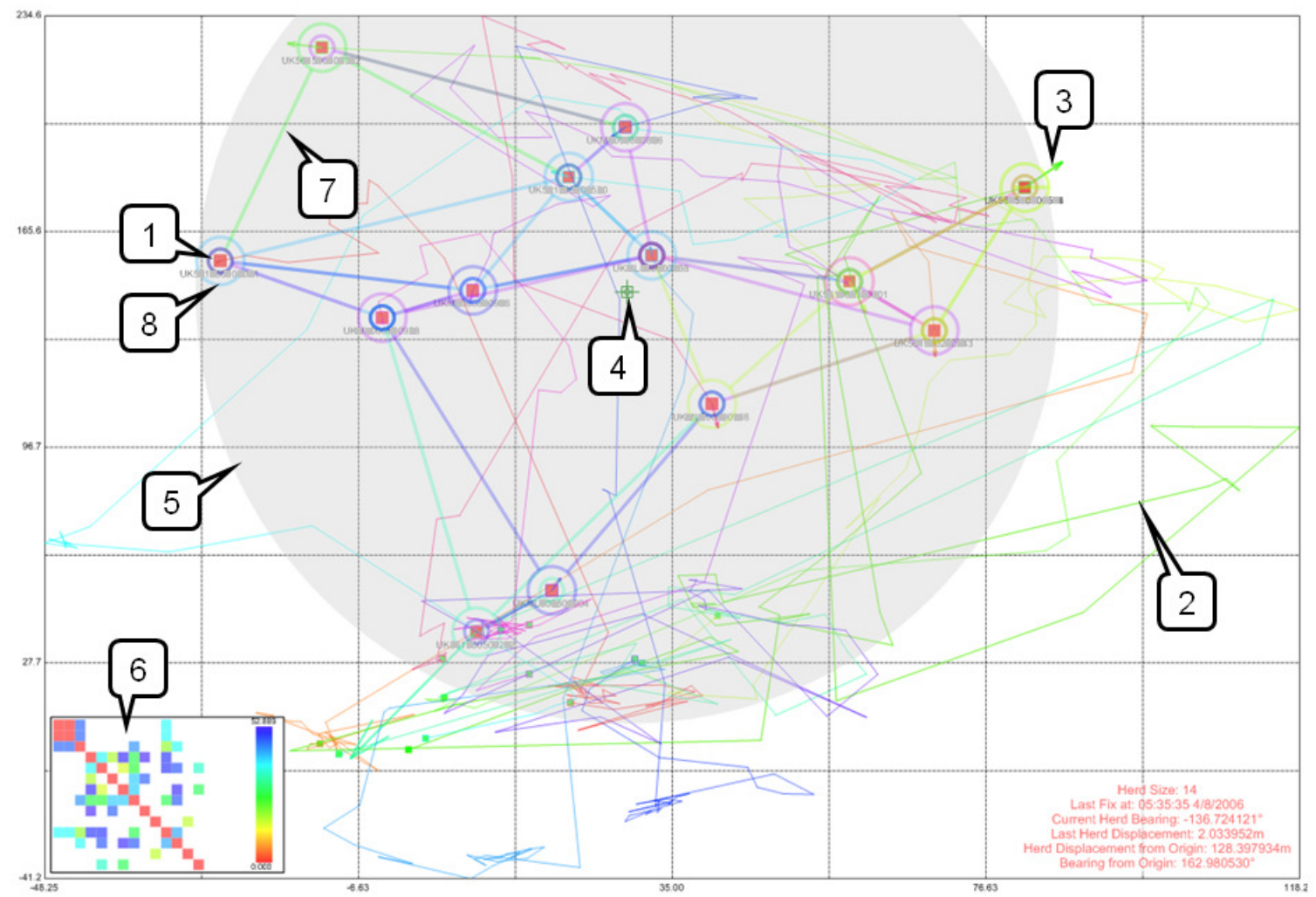

Fig. 5. The herd behavior visualization software showing model inference and salient behavioral features, specifically : $1-$ cow, $2-$ cow position trace, $3-$ bearing with magnitude denoting displacement on last fix, 4 - herd centroid, 5 - herd dispersal, 6 - inter-cow distance matrix, 7 - neighborhood connector arc, 8 - animal state (outer ring color) 
structure.

\section{Visualizing Herd Behavior}

A graphical front end to the relational database was developed to allow animated playback of the herd positions over a chosen time period and is shown in figure 5. This display not only represented the separation of the animals but also their bearings, their past positions and the magnitudes of their displacements or distance traveled. Overlaid on the graphical display were the state labels and the neighborhoods at each time step for each animal as well as the centroid and radius of dispersal for the entire herd. This facilitates interpretation of animal states and through the formation of neighborhoods allows the cliques within the herd to be easily seen. Also displayed is the inter-cow distance matrix which clearly shows the separations between animals.

\section{MODELS IN USE}

The features used in the test model were the log of the angle between the animal and herd bearings, included to capture alignment. The $\log$ of the displacement since the last fix, to capture activity levels, the $\log$ of the distance to the nearest animal and the log of the distance to the herd centroid. The last two features are used to capture solitude and cohesion respectively. Simpler models that focused on a single variable could be used to isolate particular herd traits of interest such as directional alignment or cohesion on their own rather than the approach taken here to find combinations of multiple features, which may result in less clear separation. The log scale is used to capture orders of magnitude rather than absolute values. Based on 24 hours of training data from August 2nd 2006 (midnight to midnight) the model preferred by maximum likelihood had 6 states and a neighborhood of 3 .

\section{A. Behavioral interpretation of state labels}

The mean parameters for the states of the optimal model are shown in table II and offer some degree of explanation as to what the states represent in terms of behavior. State 1 is very closely aligned with the herd bearing but exhibits a large separation from the centre of the herd and other animals in general. The displacement for animals in this state is moderate. State 2 represents animals that move much less and while close to other animals, remains distant from the centre of the herd

TABLE II

MODEL PARAMETERS

\begin{tabular}{ccccc}
\hline \hline State & $\begin{array}{c}\text { Herd } \\
\text { Alignment }\end{array}$ & Displacement & $\begin{array}{c}\text { Nearest } \\
\text { Distance }\end{array}$ & $\begin{array}{c}\text { Distance to } \\
\text { Centroid }\end{array}$ \\
\hline & & & & \\
1 & $27^{\circ}$ & $18 \mathrm{~m}$ & $15 \mathrm{~m}$ & $41 \mathrm{~m}$ \\
2 & $86^{\circ}$ & $6 \mathrm{~m}$ & $6 \mathrm{~m}$ & $15 \mathrm{~m}$ \\
3 & $119^{\circ}$ & $26 \mathrm{~m}$ & $12 \mathrm{~m}$ & $33 \mathrm{~m}$ \\
4 & $74^{\circ}$ & $9 \mathrm{~m}$ & $16 \mathrm{~m}$ & $43 \mathrm{~m}$ \\
5 & $76^{\circ}$ & $5 \mathrm{~m}$ & $9 \mathrm{~m}$ & $23 \mathrm{~m}$ \\
6 & $27^{\circ}$ & $5 \mathrm{~m}$ & $8 \mathrm{~m}$ & $20 \mathrm{~m}$ \\
\hline \hline
\end{tabular}

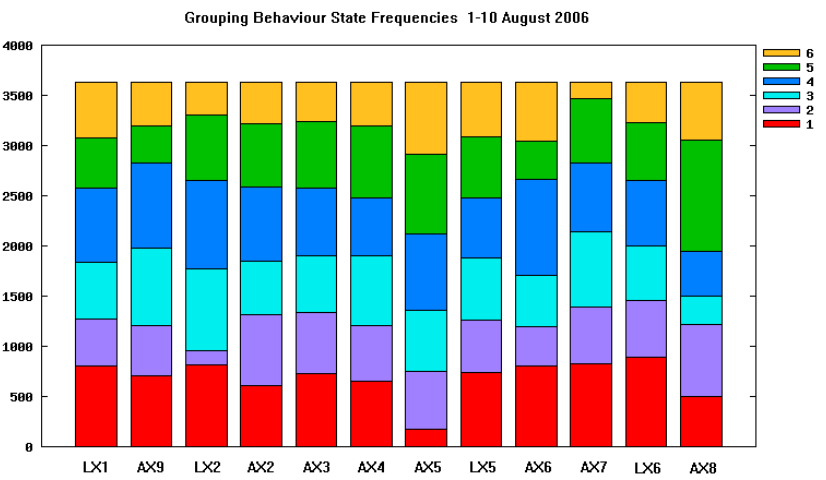

Fig. 6. Histogram of animal labeling over August $1^{\text {st }}-10^{\text {th }} 2006$.

and moves in a different direction. State 2 could be representative of a gathered clique or sub-herd while state 1 could represent a general collective movement. State 3 is not aligned with the movement of the herd at all, is separated from other animals and moves the largest distance of all animals. This behavior state may be seen as a notional 'exploratory' behavior as postulated by [6]. Similar to state 4 in terms of alignment, is state 5 - this may equate to foraging behavior or grazing again identified by $[6,8,9]$. Finally, state 6 like state 1 , represents the motion of a sub-herd albeit with more cohesion and a more sedate pace.

While visual inspection of the animated herd traces demonstrates clears separations between animals at various times of the day, a more quantifiable metric was utilized to attempt to validate state explanations. By running a trained model over 10 days of GPS traces, counts of fixes where an animal was in a particular state were accumulated into histograms an example of which is shown in figure 6. The same representation was used in [9] to produce compositions of observed daily activities. This represents the variety of behavior an individual animal exhibits over a given time, how this differs between animals and the states which dominate. For example, animal AX8 spends least time in state 3, the high displacement and unaligned state, while LX2 exhibits most occurrences of state 3 but the fewest of the more social state 2 . Animal AX5 spends little time in state 1, compared to the other animals.

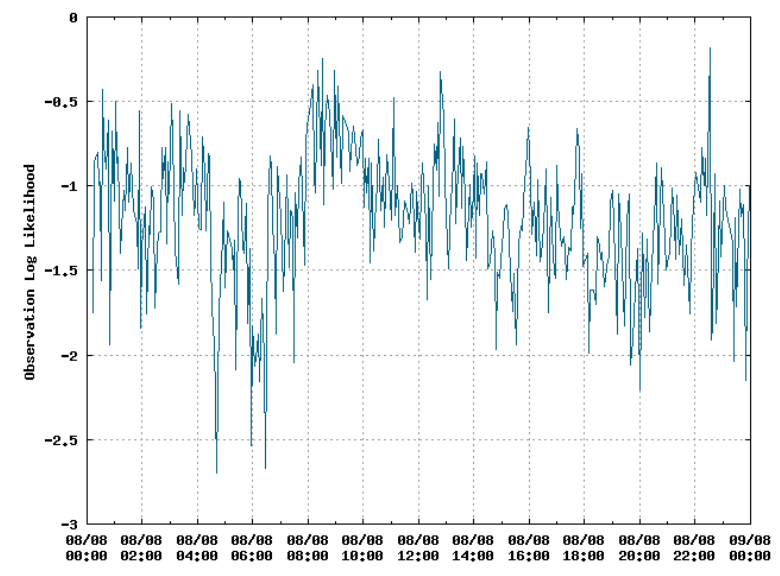

Fig. 7. Time series of model observation likelihood over August $8^{\text {th }} 2006$. 


\section{B. Indicators of Unusual Events}

As an example of identifying unusual herd configurations, 24 hours of data from August 8th 2006 was used to track the model observation likelihood over time. As figure 7 shows, the model exhibits a number of regions of low likelihood between $4 \mathrm{am}$ and $7 \mathrm{am}$. This represents a configuration that the model is unfamiliar with from the herd data it has already seen. To investigate the cause of this unlikely behavior, the animals positions were inspected manually revealing that there was a sudden large collective movement then dispersal of most animals in the herd. This was in a north easterly direction at around 4:45am. At around 6:50am most animals head south west, regrouping in the process led by animal LX5. This sequence of events may relate to the common observation that cattle outdoors tend to lie together in a group during darkness and then set of to graze or perhaps to a water source early in the morning when the sun comes up. Such collective behavior may be an evolutionary adaptation to predation during darkness.

\section{FUTURE DIRECTION}

This paper has introduced a Hidden Markov Random Field based model of group behavior and has demonstrated through position data gathered from a herd of beef cows how such a model can: identify generalized behavior of individuals and in doing so identify generalized collections of individual behaviors in groups which can then be used to track the behavior of a group with respect to its expected behavior as represented by the model through the use of the observation likelihood of the model. Through this basic functionality, it has been shown how animal behavior characteristics can be identified over a set period of time. It also demonstrated how external influences that incur extraordinary behavior can be detected as herd configurations with low likelihood. The learned models can then go on to be applied to other herds regardless of their size without having to relearn their parameters.

Animal behaviors, movements and spatial distributions can be analyzed for either individual or herd based parameters such as distance traveled, time based grazing or rumination budgets, number and duration of social interactions with other animals and consequently aspects of social status may be inferred. This paper discusses some of the ways in which GPS datasets could be analyzed and potentially modeled using machine learning approaches that would help to quantify and hence introduce some objectivity into the determination of these parameters in practical situations. Use of these modeling approaches would assist animal behavioral scientists and commercial practitioners to gain new insights into the factors which determine key features of animal performance, health and welfare under defined management or experimental conditions

The work undertaken in this paper is intended to be a first step towards using a statistical pattern recognition approach to understanding animal grouping behaviors. In the time since the trials for the work reported in this paper were completed, sensor technology has become cheaper, more reliable and the platforms used to run them e.g. [12, 13, 25] have greater computing power and programmability. Monitoring capabilities can now be expanded more easily and allow additional sensors to be incorporated into telemetry. Accurate estimation of the bearing of an animal at rest or between two very close fixes is difficult using derived quantities. Directional tracking could be improved with the inclusion of magnetometers in the collar mounted telemetry rather than relying on deriving the bearing from consecutive fixes. Similarly, close proximities may be better measured with for example, UHF proximity logging devices to alleviate potential errors from the fixes.

Hardware limitations aside, additional modeling improvements could lead to additional insight into both individual and animal grouping behavior, in particular, explicitly relating the site configurations to known herd behaviors. The model presented in this paper is a generalization of all possible behaviors seen during the training period. Segmenting this data using ground truth data such as physiological or observations of behaviors such as oestrus activity (mating behavior) into sets of distinct recognizable behaviors or interactions would allow individual models to be trained on the previously identified behaviors. The resulting ensemble could be used as a classifier for automated recognition of future occurrences of these behaviors or events. Ultimately, higher level models can be anticipated based on the one proposed here that may be used to automatically detect key management stages requiring intervention during mating periods for example, the assessment of grazing area and herd interactions during the summer grazing of environmentally sensitive grasslands, models of disease transmission in animal herds or groups, and at an individual level, behaviorally sensitive illness events.

\section{ACKNOWLEDGMENT}

The authors wish to acknowledge the help and advice over the course of this research given by Dr Rob Baxter and Alisdair Tullo of Edinburgh Parallel Computing Centre (EPCC) and Dr Hanna Miedema of the Royal (Dick) School of Veterinary Studies.

\section{REFERENCES}

[1] D. A. Wieckert, "Social Behaviour in Farm Animals", Journal of Animal Science 6:32. 1971

[2] S. A. Rands, R. A. Pettifor, J. M. Rowcliffe \& G. Cowlishaw, "State Dependent Foraging Rules for Social Animals in Selfish Herds", in Proceedings of the Royal Society 271. 2004.

[3] M.F. Bouissou, Boissy, A., Le Neindre, P. \& Veissier, I. "The social behavior of cattle" In: Social Behavior of Farm Animals (ed. L. Keeling \& H.W. Gonyou). CABI, Oxford, UK. 2001.

[4] C. W. Reynolds, "Flocks, Herds, and Schools: A Distributed Behavioral Model", Published in Computer Graphics, 21(4), pp. 25-34. July 1987.

[5] J. K. Hodgins \& D. C. Brogan, "Robot Herds: Group Behaviors for Systems with Significant Dynamics", in Proceedings of Artificial Life IV. 1994. 
[6] C. W. Reynolds, "Steering Behaviors for Autonomous Characters", in Proceedings of Game Developers Conference 1999, pp. 763-782. Miller Freeman Game Group 1999.

[7] D. Goldberg \& M. J. Mataric, "Coordinating Mobile Robot Group Behavior Using a Model of Interaction Dynamics", in Proceedings, The Third International Conference on Autonomous Agents (Agents 99), pp 100-107, ACM Press 1999.

[8] P. Juang, H. Oki, Y. Wang, M. Martonosi, L. Peh \& D. Rubenstein. "Energy Efficient Computing for Wildlife Tracking: Design and Early Experiences with ZebraNet", in Proceedings of Conference on Architectural Support for Programming Languages and Operating Systems (ASPLOS), Vol. 5, pp. 96-107, San Jose, CA, October 2002.

[9] E. Schlecht, C. Hülsebusch, F. Mahler \& K. Becker. "The use of differentially corrected global positioning system to monitor activities of cattle at pasture", Applied Animal Behavior Science 85:3-4, pp 185-202 Elsevier, March 2004.

[10] M. Schwager, D. M. Anderson, Z. Butler \& D. Rus, "Robust Classification of Animal Tracking Data", Computers and Electronics in Agriculture 56. 2007.

[11] C. M. Bishop, Pattern Recognition and Machine Learning. Springer Science + Business Media 2006

[12] T. Wark, C. Crossman, W. Hu, Y. Guo, P. Valencia, P. Sikka, P. Corke, C. Lee, J. Henshall, K. Prayaga, J. O'Grady, M. Reed \& A. Fisher, "The design and evaluation of a mobile sensor/actuator network for autonomous animal control", in IPSN '07: Proceedings of the 6th international conference on Information processing in sensor Networks, pp. 206-215, Cambridge, Massachusetts, 2007; Association for Computing Machinery, New York, NY, USA.

[13] N. Correll, M. Schwager \& D. Rus - "Social Control of Herd Animals by Integration of Artificially Controlled Congeners". Proc. of the 10th International Conference on Simulation of Adaptive Behavior (SAB). Springer Lecture Notes in Artificial Intelligence LNAI 5040 pp. 437 447, Osaka, Japan, July, 2008

[14] A Guide to Co-ordinate Systems in Great Britain, version 1.6. Ordinance Survey, Crown Copyright 1996.

[15] I. A. R. Hulbert \& J. French, "The Accuracy of GPS for Wildlife Telemetry \& Habitat Mapping", Journal of Applied Ecology 38, pp 869878. British Ecological Society 2001.

[16] G. J. McLachlan \& D. Peel, Finite Mixture Models. Wiley-Interscience. 2000.

[17] U. Grenander, Elements of Pattern Theory. John Hopkins University Press 1996.

[18] D. Mumford, "Pattern Theory: The Mathematics of Perception", in Proceedings of the International Conference on Mathematics, pp. 401422. 2002.

[19] B. S. Everitt, Introduction to Latent Class Models. Chapman and Hill, London and New York. 1984.

[20] K. P. Murphy, A Brief Introduction to Graphical Models and Bayesian Networks. http://www.cs.ubc.ca/ murphyk/Bayes/bnintro.html

[21] J. Zeng, J. \& Z. Q. Liu, "Markov Random Field-Based Statistical Modeling for Handwritten Chinese Character Recognition", IEEE Transactions on Pattern Analysis and Machine Intelligence Volume 30 No 5. May 2008.

[22] A. Dempster, N. Laird \& D. Rubin, "Maximum Likelihood from Incomplete Data via the EM Algorithm". Journal of the Royal Statistical Society, Series B. 1977.

[23] Z. Kato, T. C. Pong \& J. C. M. Lee, "Color Image Segmentation and Parameter Estimation in a Markovian Framework", Pattern Recognition Letters, 22(3-4) pp 309-321, March 2001.

[24] J. Besag, "On the Statistical Analysis of Dirty Pictures", Journal of the Royal Statistical Society. Series B (Methodological), Vol. 48, No. 3, pp. 259-302. 1986.

[25] A. Young, M. Ling \& D. K. Arvind, "Orient-2: A Wireless Real-time Posture Tracking System Using Local Orientation Estimation", in Proceedings of the 4th International Workshop on Embedded Networked Sensors (EmNets 2007), Cork, Ireland, 25-26 June 2007.

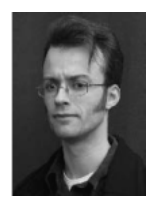

Dr Bruce Stephen currently holds the post of Research Fellow within the Institute for Energy and Environment at the University of Strathclyde. He received his BSc from Glasgow University and MSc and $\mathrm{PhD}$ degrees from the University of Strathclyde. His research interests are distributed information systems and machine learning applications in intensively condition monitored processes.

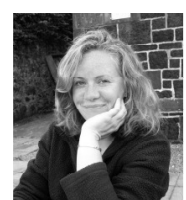

Dr Cathy Dwyer is a Reader in the Sustainable Livestock Systems Group at Scottish Agricultural College, Bush Estate. She received her BSc from University of Bristol and a $\mathrm{PhD}$ from the Royal Veterinary College, University of London. Her research interests include: mother offspring interactions, behavioural development, neonatal survival and the welfare of extensively managed farm animals.

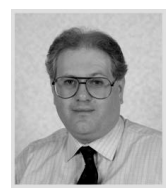

Dr Jimmy Hyslop currently holds the post of Beef Specialist within SAC's Beef and Sheep Select Group developing services to support the beef sector and helping roll out the knowledge gained from SAC research and demonstration studies. He also has a major role in conducting research and development projects for Government and commercial clients with suckler beef systems typical of those commonly found across the UK.

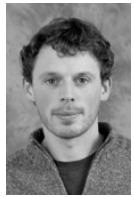

Matthew Bell is currently a $\mathrm{PhD}$ Student within the Sustainable Livestock Systems Group at Scottish Agricultural College, Bush Estate. His research interests include investigating greenhouse gas emissions for livestock and animal monitoring.

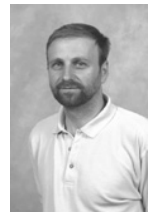

David Ross currently holds the post of Research Engineer within the Sustainable Livestock Systems Group at Scottish Agricultural College, Bush Estate. He received his BSc from Edinburgh University and PgDip from Strathclyde University. Research interests include animal monitoring for health, welfare and physiological condition and sensing systems for animal product quality.

Dr Kae Hsiang Kwong is a Research Fellow in the Centre for Intelligent Dynamic Communications at University of Strathclyde

Dr Craig Michie is a Reader within the Electronic and Electrical Engineering Department at the University of Strathclyde. He received his $\mathrm{BSc}$ and $\mathrm{PhD}$ from Glasgow University. His current research interests follow two independent themes - optical access technologies and in particular the application of semiconductor optical amplifiers (SOA) within access networks and wireless sensor systems for condition based monitoring focusing on animal monitoring.

Prof. Ivan Andonovic is the ITI Techmedia Professor of Broadband Networks and the Head of the Centre for Intelligent Dynamic Communications Systems, Strathclyde University. His research interests centre on the development of broadband networks, access and home networking and wireless sensor systems. He is Topical Editor for the 'IEEE Transactions on Communications' and was Technical Programme Co-Chair for the recent 'IEEE International Conference in Communications (ICC07)'. 